\section{ON RAPID DILATATION OF THE CERVIX UTERI.}

Read in the Section of Obstetric Medicine at the Annual Meeting of the British Medical Association, held in Glasyow, Auyust, $188 s$.

BY ALEXANDER DUKE, F.K.Q.C.I.I.,

Bix-Assistant Master jto the Rotunrla Huspital, Dublin; Gynccologrist to the Steevens's Hospital, Dubliu.

ONE of the greatest strides made by the gynacological branch of surgery has been the substitution of rapid dilatation of the cervix uteri in place of the slow, uncertain, and dangerous methods by the use of the sponge-tent or sea tangle. Tre antiseptic treatment has certainly contributed much to this advance, and has thus made the older plans of dilatation a thing of the past. We can now effect in almost as many minutes what occupied the same number of hours to accomplish previously, and I think with less pain to the patient, less trouble to the doctor, and a vast saving of time and anxiety. With a properly constructed dilator, when once carefully introduced into the cervical canal, we have the carrying out of the process of dilatation very much in our own hands, and the subsequent success of the operation depends quite as much on the judgment of the operator, and the time he takes to effect that dilatation, as in the selection of the case itself and the time chosen for interference.

I cannot but think it a great improvement to be able to accomplish with one instrument the combined work of a number, the continual introduction and withdrawal of which must prove a source of irritation even to the most tolerant uterus.

The American gynæcologists have shown that, with ordinary care, rapid dilatation is a safe operation, and can even be partially carried out in the consulting-room. Goodell, in his lectures on gynæecology, states: "For straightening out anteflexed and retruHexed uteri, and for dilating or shortening the canal in cases of sterility, or of dysmenorrhœea arising from stenosis, the clilator will be found a most efficient instrument."

Professor Munde says in his Minor Surgical Gyncecology. "Either the graduated sounds or the divergent dilators may be used, and I most decidedly prefer the divergent dilators, and have seen excellent results from their frequent und careful employment."

Dr. Ellinger, another American authority, says he has "found forcible dilatation a very efficacious remedy for flexions."

Opinions are much divided as to whether forcible dilatation or the cutting operation is the best to adopt for the cure of stenosis; for my own part I prefer the cutting operation in cases of narrow canal, conical cervix, and pinhole os, reserving the use of the dilator for the purposes of diagnosis (in cases of hiemorrhage, for instance, when the cause is doubtful), or when we wish to obtain more room in the unimpregnated organ to explore its cavity, or in cases of incomplete abortion before using the curette. The better means of access we can obtain to the uterine cavity, the better and more rapid will be our treatment of the endometrium, and instead of a long course of caustic applications, or perhaps uterine injections extending over weeks, we can remove by the curette the whole affected surface, keep the canal patulous as long as we like by a suitable flexible spiral wire stem, and so secure the recovery of the patient in one-tenth of the time required by the older methods.

I entirely agree with Dr. Goodell's statement: “I have been surprised at the improvement following a forced dilatation made in cervical endometritis for the easier introduction of remedies. - Perhaps this may be explained by the change produced in the nutrition of the parts or the temporary paralysis of the constrictor fibres, just as fissure of the anus is cured by stretching the sphincter."

The dangers of rapid dilatation of the cervix are the lighting up of old inflammatory mischief, which of course contra-indicutes its employment-cellulitis, subacute ovaritis, metritis, or even peritonitis - so that considerable caution has to be observed, the case properly selected, and the operation only undertaken by skilled hands. The uterus, as a general rule, is a most tolerant organ, but if dilatation or incision be carried to excess it will probably set up a train of symptoms analogons to those observable when laceration of the cervix has taken place during the process of labour, and may even demand a reparative operation to remedy the mischief done by a careless or too zealous practitioner.
The dilators made to divaricate by pressure on the handles are very liable to feather at the points, and unless made of a clumsythickness are bound to vield hy the pressure of the cervical canal, so that I venture to think the plun of expansion observalule in my instrument will supply the desirnd strength to effect the dilatation required, and when thus room for the introduction of the finger is gained, uny more room desired may be hal by crowding in an udditional finger, which I think will give ample room for exploration. It has struck me that a vir.w of the uterine cavity could be obtained with little trouble in these cases loy using the electric lamp, the value of which has beren recently shown in exploration of the male blachler, a much more lifficult organ to inspect than the uterine cavity.

The operation of dilatation being of short duration the exhibition of cocaine applied locally will be sulficient to keep iny severe pain in clieck, and a few drops of a 10 l"'r cent. solution injected into the cervix at each side of the os will in a short time allow interference without any complaint on the part of the patient.

The resistance felt by the operator's hand while using the -crew of my instrument will be the best guide: ats to the amount of force to be used in each case, but as a rule tr.n to fifteren minutes' time ought to suffice for obtuining suficint $\mathrm{r}(\mathrm{m}) \mathrm{m}$ for introduction of the finger, which is more than half the luttle.

It is always safer to confine the patient to bed or the recumbent posture for at least twenty-four hours after the dilatntion, though slight expansion of the cunal can be carried out in the consulting room.

My dilator can be had from Messrs. Arnold and Sons, West Smithtield, London, and has already had considerable sale.

Dr. Islacir objected to the dilating instrument exhibited hy Dr. Reid on account of its large size and it: cutting edgre. Such an instrument would require considerible and eren dangerous force to drive it through a stenosed cervis, whereas with graduated sounds force was aroided, and there was no subsequent hamorrhage.-Dr. IIErwon SNiril observed, with regard to Dr. Reid's instrument, that he fenred the screw process would injure the uterus by exercising torsion upon it; and as to Dr. Dukes dilator, he thought the divaricating blades might injure the fundus uteri. He still preferred rapid dilatation by means of craduated sounds.- Dr. AUsT laAwRENCs whjected to rapid dilatittion; he held to dilatation with tents, and proper antisejtic precautions.-IJr. BARNis concurred in the oljections to rapid dilatation, except in cases where some indication existed for reaching the carity of the uterus. He repeated his preference for incision, which not only opened up access to the uterus, but relieved congestion and inflammation which so frequently attended stenosis of the os. This dilatation could not do.Mrs. GARRYTT INDERson was surprised to hear any speak against rapid dilatation of the uterus. She was using rapid dilatation erery week, often several times a week, and she looked upon the change from the old plan of tents to the new one of rapid dilatation as one of the most solid advances: in wrinecology in recent vears.-P'rofessor STElHisson did not like the screw character of Dr. Reid's instrument-its action was clelusive. Any benefit was due to the rotatory action, not the thread. Rotation was a manceuvre adopted by all in introducing the hand or any instrument, and could be imparted to a smooth dilator--Dr. Virnnoci Cayranos agreed with the statement made by l'rofessor Stephenson, namely, that the screw was of little value, and the power of the instrument lay in the movement of rotation. If one of the instruments wis inserted into the mouth, for instance, one could easily feel that the screw did not hold, but slipped out and in as easily as a glass tube.-The President (Dr. Jore llanders) was surprised to hear, as he had just heard, that any gynacologist still preferred the old-fashioned, slow, painful, and, as he thought, comparatively unsafe method of gradual cervical dilatation with sponge or laminaria tents, to the rapid methods now arailable for this purpose. The advantages of the latter were self-evident; and whis purpose. The advantages of his own, designed for the more effectual carrying out of this process of rapid dilatation, Dr. More Madden recognised fully the excellence of the instruments just exhibited by Dr. Reid and Dr. 1)ke, both of which reflected great credit on the ingenuity of their inventors.Dr. Parvin (Philadelphin) said it seeme'd to him that, looking at these dilators, the uterus of the Britisl woman was not quite of the same form as the uterus of the American woman, for all these instruments were straight. He thouglrt that in most of the cases in which dilatation for the cure of dysmenorrhas, for example, 
was requirel, a curvel dilator was much more readily introluced. a t.1 chac was the laility most frequently used in the United states. In these cuses, certainly not very numerous, of dysmenorrhoa, where upon the introduction of a sound exquisite sensibility was found at the intermal 08, a cure was almost certain to follow the use of a curved dilator-the curved form being especially required by the fact that a flexion, usually anterior, was present. He did not believe that enlargement of the canal was in itself the curative means, at least in the majority of cases, If a computation was made of the quantity of blood that the canal must transmit in a given time, supposing the flow was of the average amount, it would only be one drop in three minutes. Surely a canal must be very small that would not permit this amount to pass without pain. So too, in some cases where the pain of menstruation was intense and the uterine sound of ordinary size could be readily passed; as it was not then enlargement of the canal, but possibly nerve-stretching, or the relief of a local inflammation, similar to the result from stretching of the anal sphincter in which painful, spasmodic contraction followed an anal fissure, that was accomplished by the use of the dilator, and thus the painful menstruation was relieved. As to the question of rapid or gradual dilatation, he certainly preferred the former, giving an anæsthetic if necessary. If dilatation was necessary for the removal of the remains of an incomplete abortion, or of an intra-uterine growth, his preference was for Hegar's dilators, or the use of sponge tents. Hegar's instruments could be made so completely aseptic, the operation could be accomplished in so short a time, and the method in all its steps could be rendered so readily and completely aseptic, that it seemed to him much better than the so-called gradual method, requiring sometimes a day or more for its accomplishment.-Dr. REID, in reply, said that he believed that in all cases of recent pregnancy, and where remains of the product of conception were retained, the finger was the best instrument both for dilatation and treatment. His dilator did not readily injure the endometrium, because the small end of the dilator projected into the cavity of the uterus. It was generally at the os internum that difficulty was found, and thus the screw had a fair hold of the tissues of the cervix below, before the point reached the difficult portion of the canal. It was a powerful dilating instrument, and therefore ought to be used cautiously and with thorough antiseptic precautions.

\section{PELVIC PERITONITIS.}

BY JAMES OLIYER, M.D., F.R.S.EDIN., Assistant Physician to the Huspital for Women, London, etc.

Pelvic peritonitis is an acute or chronic disease, consisting in inflammation affecting, it may be the whole, but more usually some part only of that serous membrane which, located within the cavity of the pelvis, enters not only into the formation of its walls and floor, but covers, more or less completely, the organs contained therein. The appellation is one, therefore, which includes peri-metritis, peri-oüphoritis, peri-salpingitis, and periproctitis. Although originating locally in the pelvis the inflammatory disturbance may, nevertheless, through a direct continuity of tissue, spread to and affect the whole peritoneal sac, constituting then what is called general peritonitis.

Inflammation of the pelvic peritoneum may occur at any period of life. It may exist before the establishment of menstruation or be developed after the cessation of the functional activity of the sexual organs. More commonly, however, it is engendered during the child-bearing epoch, the organs of generation being then more vulnerable. In the marital state the serous lining of the pelvis is especially prone to become the seat of inflammatory change, because of an increased liability to injury and disturbance of the sexual organs, consequent upon parturition and abortion. Ignorantly and unsuspiciously the woman often runs the risk of specific infection-a potent but occult influence, in many cases constantly at work, and tending to induce the disorder in question.

The infinmmatory process may result in a thickening of the peritoneun and arlhesion of opposing surfaces, the neighbouring organs, contained in the pelvis, being thereby firmly glued together. Very commonly, however, the material which exudes from the vessels, and subsequently coagulates, produces a distinct swelling, the consistence of which varies greatly. These phlegmons are often difficult to differentiate from those resulting from inflammatory exudution into the cellular tissue itself. By a process of fatty degeneration and liquefaction the plastic exudation occurring in pelvic peritonitis may eventually become absorbed. Adhesions, however, generally ensue, the existence of which may be indicated by a more or less marked deviation of the uterus, the organ, that is, being more or less closely applied to one or other wall of the pelvis. Effusion of serum may take place, and this, when confined and large in amount, or rather, when capable of detection, constitutes what is termed encysted serous peritonitis. The transition from that of a serous to a purulent effusion is a gradual one. In the latter case the pus corpuscles are merely far more abundant than in the former. When effusion of serum has occurred the liquid portion may at length completely disappear by a process of gradual absorption. At first absorption takes place, as a rule, somewhat rapidly; as concentration advances, however, it is much more slowly effected. After absorption the disordered peritoneal surfaces come in contact and adhere. When the exudation is purulent this, too, may eventually become absorbed-the serous portion directly, the fibrin and pus after undergoing a fatty metamorphosis. This, however, comparatively speaking, rarely happens.

In pelvic peritonitis small suppurations frequently take place, and these, as distinct abscess formations, cannot be detected by the most careful examination. The probable existence of such in. any given case is only surmised when small quantities of pus are. detected with the rectal evacuations. Fortunately these ruptures occur most commonly into the gut ; they may, however, as I have occasionally witnessed, rupture into the peritoneal sac, and so cause death. Regarding the mode of formation of these small suppurations, I have a word to say. They appear to me to resuls from the generation of pus corpuscles in the tissue of the peritoneum itself, and the perforation results merely from the deep penetration of these cells.

As to the etiology of pelvic peritonitis I have but little definite information to impart. The disease is especially rife in women, and may be considered peculiar to the female habit of body. This. to my mind, apart altogether from the risks incidental to child-bearing, or those dependent to a certain extent upon anatomical construction and direct structural continuity, points to a marked disposition on the part of this serous membrane in the female to inflammatory change.

Pelvic peritonitis is frequently idiopathic, and in many cases the exciting causes are not to be explained. The disease often develops in persons already debilitated or in association with some other disorder; this, however, proves no dependence, and does not. justify the conclusion that the disease is secondary. Under such circumstances it ought to be viewed rather as a complication. Peritonitis accompanying puerperal fever and other infectious disorders may, however, be considered secondary. Injury is an exciting cause, and this need not be severe, as some persons seem especially prone to this inflammatory disease. I have seen fatal peritonitis follow upon the most trifling interference with the uterus, and this accident, when it occurs, is a most unfortunate one, apt as it is to engender invective. A most careful and thorough examination of the pelvic organs should be effected prior to undertaking any surgical or other interference with the uterus. Fven in the hands of the most skilled gynacologist this task is not always an easy one, as it is often most difficult to detect slight adhesions.

Pelvic peritonitis may he induced by a direct extension of inflammatory disturbance through continuity or contiguity of tissue, as happens frequently in salpingitis, especially when this is the result of gonorrhmal infection.

Inflammation of the serous lining of the pelvis may also be caused by neoplasms approaching the peritoneal surface, is happens frequently when the growth is malignant, and much more rarely when it is benign.

In many cases this disorder appears to result from exposure to cold, from the indiscriminate use of medicated injections, and the adoption of other ill-advised methods of protection against impregnation. It may also be caused by the rupture into the peritoneum of a cyst (containing virulent fluid), or abscess of the ovary or of any other structure. Such abscesses may be simple or tubercular. It sometimes results from the discharge of any irritant fluid into the peritoneal sac, either through an abnormal or. it may be, the natural opening of the Fallopian tube, hence the risk in injecting simple or medicated fluids into the cavity of the uterus.

Pelvic peritonitis, it is alleged, is more common in primipare 